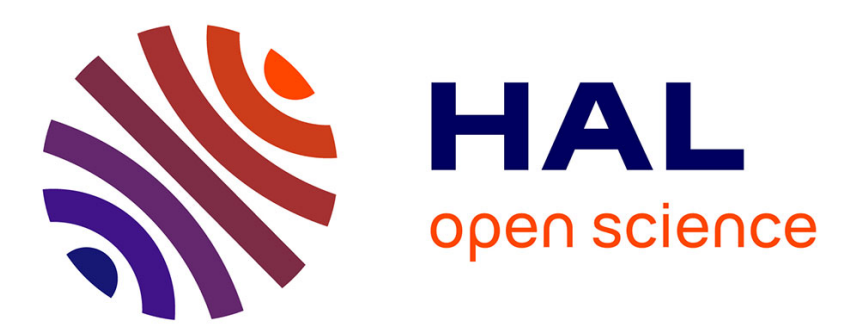

\title{
Francophone Muslim intellectuals, Islamic associational life and religious authority in Burkina Faso
}

Frédérick Madore

\section{To cite this version:}

Frédérick Madore. Francophone Muslim intellectuals, Islamic associational life and religious authority in Burkina Faso. Africa, 2020, 90 (3), pp.625-646. 10.1017/S0001972020000108 . halshs-02614959

\section{HAL Id: halshs-02614959 \\ https://shs.hal.science/halshs-02614959}

Submitted on 21 May 2020

HAL is a multi-disciplinary open access archive for the deposit and dissemination of scientific research documents, whether they are published or not. The documents may come from teaching and research institutions in France or abroad, or from public or private research centers.
L'archive ouverte pluridisciplinaire HAL, est destinée au dépôt et à la diffusion de documents scientifiques de niveau recherche, publiés ou non, émanant des établissements d'enseignement et de recherche français ou étrangers, des laboratoires publics ou privés. 


\title{
Intellectuels musulmans francophones, vie associative islamique et autorité religieuse au Burkina Faso
}

\author{
Frédérick Madore ${ }^{1}$
}

\section{Introduction}

Depuis la chute du président Blaise Compaoré le 31 octobre 2014 à la suite d'une insurrection populaire qui a mis fin à ses 27 ans de règne, la radicalisation islamique et les épisodes de violence ont marqué l'actualité du Burkina Faso, pourtant souvent présenté comme un modèle de coexistence pacifique entre les religions (Langewiesche 2011; Audet Gosselin 2016). Longtemps épargné par les groupes armés du Sahel, ce pays se trouve dorénavant au cœur des troubles de la région avec une multiplication d'attentats terroristes depuis 2016 (International Crisis Group 12 octobre 2017; Nsaibia 2018; Nsaibia et Caleb 2018). À première vue, les menaces sécuritaires et les actions d'Ansaroul Islam - première organisation djihadiste de l'histoire du pays - ont laissé croire à une radicalisation de certains cercles musulmans. Cependant, l'attention médiatique portée sur ces craintes et les nombreuses analyses sur l'extrémisme au Burkina Faso, bien qu'elles soient légitimes, ont éclipsé des transformations sous-jacentes qui sont en train de s'opérer dans le paysage associatif islamique depuis l'insurrection populaire d'octobre 2014. En effet, ce bouleversement politique majeur a eu des conséquences significatives sur la participation des musulmans dans les débats sociopolitiques, les dynamiques intergénérationnelles et, plus largement, les bases sur lesquelles l'autorité religieuse est revendiquée.

Cet article propose donc d'analyser la concurrence que se livrent les acteurs islamiques dans la sphère publique pour le leadership religieux au Burkina Faso depuis 2014 à travers trois axes : quels sont les fondements sur lesquels s'appuient les organisations islamiques et leurs responsables pour revendiquer le droit de s'exprimer au nom de « la communauté musulmane » du pays? Quelle est l'influence de l'État sur l'autorité religieuse par la désignation des interlocuteurs privilégiés dans ses rapports avec les musulmans. Enfin, quelle

\footnotetext{
${ }^{1}$ Frédérick Madore est chercheur postdoctoral Banting au Center for African Studies à l'University of Florida (États-Unis) et membre du Sahel Research Group. Il est notamment l'auteur de La construction d'une sphère publique musulmane en Afrique de l'Ouest (Presses de 1’Université Laval/Hermann, 2016). Email: frederick.madore@ufl.edu
} 
est la capacité d'individus et de groupes appartenant à des catégories sociales d'ordinaire marginalisées comme les jeunes à émettre publiquement des opinions divergentes par rapport aux discours des représentants « officiels » ou « autoproclamés » de leur propre communauté?

À cet égard, les développements récents au Burkina Faso font écho à la diversification des types et des sources d'autorité religieuse visible ailleurs dans le monde musulman contemporain. De plus en plus d'individus et de groupes de différents segments de la société réclament aujourd'hui le droit de s'exprimer au nom de l'islam (Eickelman et Piscatori 2004; Krämer et Schmidtke 2006; Mandaville 2007), dont de nouveaux types d' « intellectuels », qui jouent un rôle majeur dans la sphère publique (Bamyeh et Salvatore 2017). À l'image des « nouveaux clercs séculiers de l'islam » incarnés par l'intellectuel ou le « conférencier » musulman (Frégosi 2004), l'autorité religieuse que revendiquent des leaders musulmans francophones au Burkina Faso découle moins d'un savoir religieux classique ( 'ilm) que d'une capacité à s'engager dans la sphère publique pour débattre des notions de « bien commun »l'al-mașlaḥa al- 'amma (Salvatore et Eickelman 2006).

L’implication accrue au cours des dernières années de figures musulmanes burkinabè dans l'arène sociopolitique contraste nettement avec le large consensus qui s'est dégagé parmi la production scientifique quant à la spécificité de l'islam au Burkina Faso par rapport aux pays de la sous-région. Dans son étude comparative sur le Sahel, Idrissa (2017) a affirmé que l'apolitisme et la passivité (« quiescence ») de l'islam au Burkina Faso représentaient un cas d'exception. À l'instar des travaux qui ont souligné la subordination politique et la cooptation des élites musulmanes burkinabè (Otayek 1984, 1996; Cissé 1994; Kouanda 1998; Vanvyve 2015; Madore 2016a: 46-55; 70-91), Samson a avancé que « l'islam au Burkina Faso n'est pas inscrit dans une démarche de politisation » et qu'il «n'a jamais représenté une force contestataire » (2018: 292-293). Bien que l'islam soit devenu la religion majoritaire ${ }^{2}$, c'est

\footnotetext{
${ }^{2}$ La proportion de musulmans a plus que doublé en un demi-siècle : selon un recensement de 1960-1961, la Haute-Volta comptait 27,5 \% de musulmans (contre 68,7 \% d'animistes et $3,7 \%$ de catholiques); selon des chiffres du dernier recensement en 2006, les musulmans représentaient $60,5 \%$ de la population contre $23,2 \%$ de chrétiens. Voir 'Enquête démographique par sondage en République de Haute-Volta 1960-1961 (Tome I)', 〈www.odsef.fss.ulaval.ca/sites/odsef.fss.ulaval.ca/files/fonds_gp/c-doc_365_odsef.pdf $>$,
} 
plutôt l'Église catholique qui, à travers son vaste réseau d'écoles implanté dès la période coloniale, s'est assuré le quasi-monopole de l'élite dirigeante de l'État postcolonial burkinabè (Otayek 1997; Somé 2001; Bouron 2011). Encore aujourd'hui, les catholiques se perçoivent comme une minorité religieuse dominante en raison de leur poids dans l'appareil étatique et du prestige de leurs établissements éducatifs et sanitaires (Kane 2016), et ce, malgré l'essor des mouvements pentecôtistes et évangéliques ainsi que du renouveau charismatique catholique depuis les années 1990 (Laurent 2009 [2003]; Fancello 2007).

La présente recherche se base sur deux enquêtes de terrain conduites à Ouagadougou en 2011 et 2015. Des entretiens ont été menés auprès de dirigeants et de militants de la plupart des principales associations islamiques du pays ${ }^{3}$. Le choix de se concentrer sur ces organisations nationales s'explique par le fait qu'elles sont un véhicule privilégié pour les musulmans qui souhaitent investir la sphère publique et prendre position dans les débats sociopolitiques. Outre certaines grandes figures religieuses telles que les imams ou les prêcheurs, ce sont généralement les responsables et les porte-paroles des associations islamiques nationales qui sont les plus médiatisés. L'analyse se focalise principalement sur la perspective des « intellectuels musulmans » francophones, qui gravitent autour de l'Association des Élèves et Étudiants Musulmans au Burkina (AEEMB) ${ }^{4}$ et du Cercle d'Études, de Recherches et de Formation Islamiques (CERFI) ${ }^{5}$. Au Burkina Faso, tout un imaginaire s'est construit autour

consulté le 29 janvier 2018 et 'Recensement général de la population et de l'habitation au Burkina Faso (RGPH) en 2006', <www.insd.bf/n/index.php/publications/18-lespublications/enquetes-et-recensements/141-recensement-general-de-la-population-et-de-1habitation-au-burkina-faso-rgph-en-2006> , consulté le 20 janvier 2018.

${ }^{3}$ Association des Élèves et Étudiants Musulmans au Burkina (AEEMB), Cercle d'Études, de Recherches et de Formation Islamiques (CERFI), Communauté Musulmane du Burkina Faso (CMBF), Fédération des Associations Islamiques du Burkina (FAIB), Ittihad Islami et Mouvement Sunnite.

${ }^{4}$ Officiellement reconnue en 1986, l'AEEMB vise la promotion de l'islam dans le milieu éducatif francophone. L'association a été active de manière informelle sous d'autres noms à partir des années 1970 : Union Discipline Croyance, Troupe Muhammad et Association Musulmane des Scolaires Voltaïques.

${ }^{5}$ Le CERFI, créé en 1989, est en quelque sorte le prolongement de l'AEEMB pour les militants qui ont intégré le marché du travail. 
du statut d' « intellectuel » pour désigner les étudiants et diplômés universitaires

(Mazzocchetti 2009). Ainsi, par extension, les musulmans, qui ont suivi un cursus scolaire francophone, laïque ou chrétien, et qui ont intégré la fonction publique ou le secteur économique officiel, se qualifient d' «intellectuels musulmans ». Cette catégorie, qui revêt une acceptation large parmi ces acteurs, exclut généralement les arabisants formés dans les universités arabes. Plusieurs travaux ont d'ailleurs souligné l'importance de ces musulmans francophones, qui interviennent de plus en plus dans la sphère publique pour prendre position sur des enjeux sociaux et prescrire des normes morales au Burkina Faso (Saint-Lary 2011; Ouédraogo 2019; Madore 2016a: 151-61, 2016b) et ailleurs en Afrique de l'Ouest (MiranGuyon et Oyewolé 2015; Sounaye 2015; Camara et Bodian 2016).

L'étude repose aussi sur le dépouillement d'articles de journaux de la presse généraliste et islamique pour relever les prises de position publiques d'acteurs musulmans du Burkina Faso pour lesquels l'article laisse une grande place. La presse généraliste est également très révélatrice de l'influence médiatique, de l'importance relative et du traitement dont bénéficient différentes figures et associations islamiques. Elle s'avère donc utile pour examiner la participation de groupes qui sont habituellement exclus des débats publics dominants et les rapports de pouvoir qui y sous-tendent.

Dans la première partie, un retour sur le paysage associatif islamique sous l'ère Compaoré permettra de montrer que le fossé entre la gérontocratie aux commandes des grandes associations islamiques du Burkina Faso et les jeunes, qui s'était creusé tout au long des décennies 1990 et 2000, se révéla avec force suivant le soulèvement populaire d'octobre 2014. De nombreux musulmans, dont des jeunes, qui étaient auparavant très réticents à critiquer ouvertement la conduite des représentants de leur communauté, dénoncèrent sévèrement leur soutien plus ou moins tacite envers l'ancien régime. Ceci amena les responsables de la Fédération des Associations Islamiques du Burkina (FAIB) à confier une plus grande place aux jeunes et aux intellectuels musulmans dans la direction de l'organisation.

La seconde partie analysera différents discours et diverses initiatives d'acteurs musulmans, qui sont évocateurs d'un désir de s'impliquer plus directement dans les débats nationaux afin de se positionner plus avantageusement dans le champ islamique burkinabè. Profitant de l'espace laissé vacant par les porte-paroles traditionnels de la communauté durant le processus de transition, certains jeunes « intellectuels musulmans » francophones cherchèrent 
activement à se présenter comme les véhicules d'un « islam civil » (Hefner 2011 [2000]) en promouvant de nouvelles formes d'engagement citoyen par le religieux. S'ils n'ont pas remplacé les grandes autorités religieuses du pays, qui continuent d'exercer une influence considérable, cette élite est néanmoins parvenue à faire de l'AEEMB et surtout du CERFI des intermédiaires privilégiés entre les musulmans et le gouvernement du président Roch Marc Christian Kaboré. D'autres organisations musulmanes tentèrent également de tirer profit du nouveau contexte politique pour occuper plus activement l'arène sociopolitique comme en témoignèrent les prises de position des porte-paroles de la FAIB lors des élections de 2015, puis concernant le projet de loi sur les libertés religieuses en 2017.

\section{D'un changement de garde politique à un nouveau leadership islamique? Turbulences au sein de la FAIB}

Tout au long de sa présidence, Blaise Compaoré s’appuya avec succès sur un modèle néopatrimonial classique alliant cooptation et clientélisme à l'endroit des élites musulmanes afin de s'assurer de leur soutien. La figure d'Oumarou Kanazoé, qui cumulait d'importantes fonctions économiques et politiques en plus de celle de guide religieux, fut centrale dans cette entreprise. Les frustrations croissantes d'une partie de la jeunesse musulmane à l'égard de la gérontocratie à la tête de leur communauté religieuse, qui étaient restées latentes, s'exprimèrent ouvertement dans les mois suivant l'insurrection populaire. Ceci entraina de premiers changements notables au sein de la FAIB.

Les associations islamiques sous le régime Compaoré : des logiques clientélistes et gérontocratiques sur fond de tensions intergénérationnelles

Au début des années 1990, suivant l'adoption de programmes d'ajustement structurel promus par le Fonds Monétaire International et la Banque mondiale, le pouvoir Compaoré lança un vaste plan de privatisation des entreprises publiques. Ceci offrit des perspectives d'enrichissement importantes pour les acteurs économiques locaux. Cependant, l'accès à ces opportunités fut conditionné à la proximité avec le parti au pouvoir, l'Organisation pour la Démocratie Populaire-Mouvement du Travail (ODP-MT) - devenu le Congrès pour la Démocratie et le Progrès (CDP) en février 1996 -, qui avait rapidement étendu ses tentacules aux secteurs clés de l'économie burkinabè (Harsch 2017: 140-158).

Dans ce contexte, nombre d'entrepreneurs et de commerçants musulmans, qui occupaient une position prépondérante dans plusieurs secteurs de l'économie burkinabè, travaillèrent plus ou 
moins ouvertement à soutenir le régime en place pour favoriser la prospérité de leurs affaires. Beaucoup de ces acteurs s'adonnaient à diverses activités de mécénat religieux si bien qu'ils exerçaient une forte influence auprès de leurs coreligionnaires (Cissé 2015: 16-17). Ainsi, certains d'entre eux purent mettre à profit leur capital financier pour s'imposer dans plusieurs des grandes associations islamiques du pays même s'ils n'avaient pas d'études religieuses formelles. Au Mouvement Sunnite, alors que des musulmans francophones avaient joué un rôle central dans la création de la structure en 1973 et en avaient assuré la direction pendant plus de vingt ans (Cissé 2009; Kobo 2012), les militants se tournèrent vers de riches entrepreneurs économiques peu instruits pour la présidence de l'organisation à partir de $2001^{6}$.

L'entrepreneur Oumarou Kanazoé, grand «mécène de la communauté musulmane » (Cissé 2010), incarna plus que quiconque l'accord tacite entre la classe politique et économique ainsi que cette influence de plusieurs acteurs économiques auprès des milieux islamiques. Ayant amassé une fortune colossale à partir des régimes précédents, il devint président de la Chambre de commerce et d'industrie du Burkina Faso en novembre 1995 et du Conseil national du patronat burkinabè en 1997. En plus de ces deux postes qu'il occupa jusqu'à sa mort en octobre 2011, il fut également un important collecteur de fonds et donateur pour le parti de Blaise Compaoré. En 2007, Kanazoé fut choisi président d'honneur de la Fédération associative pour la paix et le progrès avec Blaise Compaoré (FEDAP-BC), une « organisation de la société civile » qui « entend fédérer les forces de tous les admirateurs du chef d'État burkinabè » (Sidwaya 16 octobre 2007).

Parallèlement à ses fonctions économiques et politiques, Kanazoé était aussi très influent auprès de ses coreligionnaires. Tout au long de la grave crise interne au sein du Mouvement Sunnite entre 1995 et 2006 - qui avait entrainé la suspension de l'association et la fermeture à deux reprises de la grande mosquée de Zangouettin à Ouagadougou (Cissé 2009: 18-28) -, il avait assuré la médiation entre le Ministère de l'Administration Territoriale et les protagonistes. Kanazoé exerçait surtout un poids considérable sur la Communauté

\footnotetext{
${ }^{6}$ Aboubacar Compaoré, un transporteur qui ne sait lire ni le français ni l'arabe, a assuré la présidence du Mouvement Sunnite entre 2001 et 2007. Adama Nikiéma, l'actuel président en poste depuis 2007, est un commerçant de Ouagadougou, qui est lui aussi analphabète.
} 
Musulmane du Burkina Faso (CMBF) qui, depuis sa création en $1962^{7}$, était considérée comme une des trois principales interlocutrices des musulmans auprès de l'État aux côtés du Mouvement Sunnite et de la Communauté Islamique de la Tijâniyya du Burkina Faso $(\mathrm{CITBF})^{8}$. Les journalistes affectés à la couverture du congrès de la CMBF en 1997 avaient souligné qu'Aboubacar Sana, le nouveau président de l'association, avait pu compter sur l'appui de Kanazoé. Ce soutien était de taille puisque ce dernier avait couvert l'entièreté des dépenses des cinq cents congressistes (Sidwaya 24-25 décembre 1997). En 2004, Kanazoé fut porté à la direction de la CMBF, poste qu'il avait déjà assuré entre 1977 et 1982. À l'instar du Mouvement Sunnite, le choix de cet homme d'affaires analphabète consacra le déclin des musulmans francophones issus de l'administration publique dans la direction de la CMBF, qui y occupaient auparavant une place centrale, et ce, dès les années $1960^{9}$.

En décembre 2005, Kanazoé prit également la tête de la nouvelle Fédération des Associations Islamiques du Burkina (FAIB) pour laquelle il avait personnellement supporté les coûts du congrès constitutif de cette structure, qui réunissait alors plus d'une centaine d'associations islamiques, dont la totalité des plus importantes du pays ${ }^{10}$. Si l'idée d'une telle organisation remontait aussi loin que dans les années $1980^{11}$, le processus s'était surtout mis en branle à partir du début des années 2000 suivant le constat de la prolifération presque anarchique des groupes se réclamant de l'islam au Burkina Faso ${ }^{12}$. Un des objectifs de la FAIB est d'ailleurs

\footnotetext{
${ }^{7}$ Anciennement la Communauté Musulmane de Haute-Volta (CMHV).

${ }^{8}$ Anciennement l'Association Islamique de la Tijâniyya (AIT), créée en 1978.

${ }^{9}$ Toumani Triandé en est un exemple éloquent. Nommé secrétaire général de l'association en 1962, cet instituteur de formation, qui fut notamment muséologue, député et ministre, présida la CMHV/CMBF de 1981 à 1983 et de 1986 à 1997.

${ }^{10}$ Entretien avec des responsables de la FAIB, Ouagadougou, 28 avril 2015.

${ }^{11}$ Entretien avec Souleymane Ouédraogo, ancien président du Mouvement Sunnite (19731988), Ouagadougou, 5 novembre 2011.

${ }^{12}$ Entretien avec des responsables de la FAIB, Ouagadougou, 28 avril 2015.
} 
de « Représenter les associations membres sur le plan national et offrir à la communauté des musulmans du Burkina un porte-parole unique ${ }^{13} »$.

Bien que l'AEEMB et le CERFI fassent partie des six associations fondatrices, ils furent exclus du «présidium » de la FAIB constitué des présidents de la CMBF, du Mouvement Sunnite, de la CITBF et d'Ittihad Islami, qui disposent d'un droit de veto. Même si des individus gravitant autour de l'AEEMB et du CERFI avaient activement plaidé pour l'unité de la oumma burkinabè dès les années 1990 et avaient entrepris de premières démarches concrètes à partir de $2001^{14}$, des représentants de ces deux organisations durent se contenter de la sixième et septième vice-présidence de la FAIB en plus de quelques postes d'adjoints ${ }^{15}$. En dépit du fait que des membres du CERFI aient « estimé qu'on leur a confisqué la structure pour aller dans une orientation qui n'était pas celle du départ» selon un livre produit par cette association (Sawadogo, Gansonré et Ouibga 2016: 23-24), ses dirigeants préférèrent ne pas mettre en péril la fédération et, ce faisant, l'unité de la communauté.

Selon Aboubacar Doukouré, membre du présidium de la FAIB, la présidence fut confiée à Kanazoé «parce que c'était une personnalité incontournable, parce qu'il est respecté par tout le monde. Avec son âge d'abord parce qu'il a plus de 80 ans. Donc personne parmi nous ne peut rivaliser. Avec ses bienfaisances parce qu'il n'y a pas un chef religieux dans notre pays ou bien une association islamique que Kanazoé n'a pas rendu service. [...] Parce qu'avec les jeunes ça pose des problèmes. Quand on dit untel est président, les autres vont contester ${ }^{16}$. »

13 'Récépissé de déclaration d'association n²006-078/MATD/SG/DGLPAP/DOASOC du 07 mars 2006', <www.legiburkina.bf/m/Sommaires_JO/Récépissé_MATD_2006_00078.htm>, consulté le 25 novembre 2017.

${ }^{14}$ En juin 2001, des membres du CERFI avaient organisé la « Conférence des Cadres et Intellectuels Musulmans du Burkina », qui se voulait un forum pour jeter les bases d'une « unité organisationnelle » des musulmans du pays, auquel étaient conviés les responsables de 85 associations islamiques (Sidwaya 19 juin 2001).

15 'Récépissé de déclaration d'association n²006-078/MATD/SG/DGLPAP/DOASOC du 07

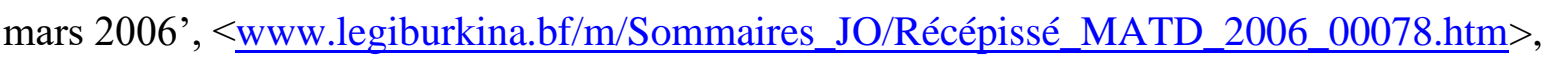
consulté le 25 novembre 2017.

${ }^{16}$ Entretien avec Aboubacar Doukouré, fondateur et guide spirituel d'Ittihad Islami et membre du présidium de la FAIB, Ouagadougou, 15 avril 2015. 
Ces propos illustraient bien l'importance du capital financier, mais aussi le caractère grandement gérontocratique du leadership de la communauté musulmane au Burkina Faso, que nous avait ainsi résumé un jeune musulman : « les responsables [des principales associations islamiques], ce sont les vieux et les riches. Tu es jeune, tu n'as pas ta place $^{17} \gg$. Pour le président du CERFI entre 2009 et 2015, l'implication des jeunes « intellectuels » de son association au sein de la FAIB était « difficile»: « On essaie d'être présent parce qu'on pense qu'on a beaucoup de choses à apporter [...]. On ne peut pas changer les choses d'un coup de baguette magique. Il faut aller au rythme de tout le monde ${ }^{18}$. »

D'autres voyaient aussi le travail du pouvoir derrière la nomination de Kanazoé afin de mieux domestiquer l'islam et ses principaux représentants. Toujours est-il que les liens clientélistes étroits unissant Kanazoé au régime, à peine masqués, expliquaient bien pourquoi les leaders des principales organisations islamiques demeurèrent inféodés à l'État tout au long du règne de Compaoré. En effet, sous leur apolitisme de façade, ils contribuèrent surtout à la légitimation du pouvoir en faisant preuve de complaisance à l'égard de ses dérives et en cherchant, de leur propre initiative ou suivant une sollicitation de l'État, à éteindre toute mobilisation contestataire potentielle des fidèles musulmans. Le cas de la mort du journaliste Norbert Zongo en décembre 1998 l'illustra bien. De nombreux Burkinabè y virent un assassinat orchestré par le régime, car il avait entamé une enquête sur le rôle du « petit président » François Compaoré - le frère cadet du président et son conseiller - dans le décès de son chauffeur. Alors que le président Compaoré faisait face à sa plus grave crise sociopolitique depuis son arrivée au pouvoir, les responsables de neuf organisations musulmanes parmi les plus importantes du pays invitèrent la population à préserver l'ordre et la paix dans une lettre ouverte (L'Observateur Paalga 4 janvier 1999). Un autre exemple est la participation des leaders musulmans lors de la Journée Nationale du Pardon (JNP) ${ }^{19}$ en mars 2001 (Vanvyve 2015: 496-500).

\footnotetext{
${ }^{17}$ Entretien avec un ancien militant de l'AEEMB, Ouagadougou, 24 avril 2015.

${ }^{18}$ Entretien avec Moussa Nombo, ancien président du CERFI (2009-2015), Ouagadougou, 16 juin 2015.

${ }^{19}$ Cet évènement, placé sous l'égide des autorités religieuses et coutumières ainsi que d'anciens chefs d'État du pays, se voulait un mea culpa national pour tous les crimes politiques commis depuis l'indépendance - les plus notables étant les assassinats de Thomas
} 
Même si ces leaders optèrent souvent pour des positions en décalage avec l'insatisfaction croissante de la population et notamment d'une partie de la jeunesse musulmane à l'égard du régime Compaoré, les jeunes furent pour la plupart caractérisés par leur mutisme presque complet quant à la conduite de leurs ainés. Beaucoup de jeunes musulmans avec lesquels nous nous sommes entretenus accordent une grande importance au respect traditionnel du statut associé à l'ainesse - les « parents »-, de sorte que nombre d'entre eux se montrèrent réticents à les critiquer ouvertement malgré les désaccords. En effet, la culture traditionnelle moaga, prépondérante à Ouagadougou, s'avère extrêmement hiérarchisée et gérontocratique. Le respect de l'autorité des ainés a une grande incidence sur la prise de parole des cadets (Gruénais 1985; Laurent 2009: 263-269). Plus largement, la société burkinabè sous la présidence de Compaoré fut marquée par une fracture générationnelle, qui s'exprimait à travers la domination politique, sociale et économique des ainés sur les jeunes en dépit de leur poids démographique considérable (Hilgers et Loada 2013: 203-205). Les propos tenus par Issaka Sam, président du CERFI entre 1991 et 1997, furent particulièrement évocateurs du respect accordé aux « anciens » :

[...] nous ne sommes pas de ceux qui repoussent les anciens. Les anciens ont beaucoup fait. Ce sont eux qui ont été les gardiens de la religion. Nous essayons, nous les jeunes, d'avoir de très bonnes relations avec eux et les amenons petit à petit à comprendre les changements qu'il y a dans ce monde. [...] Il faut essayer de s'adapter. Nos rapports sont donc des rapports de respect, nous reconnaissons aux anciens leurs droits d'aînés et nous souhaitons pouvoir travailler toujours ensemble parce que ni eux ni nous les jeunes ne pouvons à nous seuls apporter un changement positif. (L’Observateur Paalga 5 février 1997)

Les tensions intergénérationnelles au sein de la communauté demeurèrent par conséquent essentiellement larvées tout au long des années 1990 et 2000. Ainsi, aucun leader de l'AEEMB et du CERFI n'avait précisé publiquement leur position sur la situation nationale suivant la mort de Norbert Zongo. Ils étaient également restés silencieux sur la participation de plusieurs de leurs ainés à la JNP deux ans plus tard. Cependant, la fracture générationnelle

Sankara et du journaliste d'investigation Norbert Zongo - sans établir de coupables pour ces méfaits. Aboubacar Sana, Aboubacar Doukouré et Aboubacar Maïga II furent les trois représentants des musulmans dans le présidium de la JNP. 
s'était accentuée en 2013 dans le cadre de la mise en place d'un Sénat. L'opposition estimait que le président Compaoré avait l'intention de s'en servir pour réviser l'article 37 de la constitution, qui l'empêchait de se présenter à l'élection de $2015^{20}$. La sortie publique du secrétaire général de la FAIB en septembre pour endosser le projet d'instauration d'une chambre haute au nom de la « communauté musulmane » avait contribué à l'insatisfaction grandissante envers les responsables de la fédération dans un contexte où le régime Compaoré devenait de plus en plus désavoué ${ }^{21}$. D'ailleurs, la centaine de commentaires suscitée par la nouvelle du soutien de la FAIB au Sénat sur Burkina24 ${ }^{22}$ et LeFaso.net ${ }^{23}$ témoignait bien de la crise de légitimité de l'organisation au sein d'une partie de la population et des musulmans eux-mêmes. En effet, des internautes, dont plusieurs se disant musulmans, avaient vilipendé les dirigeants de la FAIB et tout spécialement son porte-parole, qui faisait « honte à l'islam » et « gâtait le nom des musulmans ». Certains avaient présenté les responsables de la fédération comme des commerçants analphabètes guidés par leur ventre.

L'AEEMB et le CERFI s'étaient même distancés très clairement de la position de la FAIB dans une lettre signée par leurs présidents et publiée dans les journaux burkinabè quelques jours plus tard (Madore 2016b: 17-21). Néanmoins, l'exacerbation des tensions intergénérationnelles n'avait pas encore conduit à une contestation ouverte. Comme l'a souligné Vanvyve, malgré cet épisode, le « respect de l'aîné semble difficilement contournable » pour nombre de jeunes musulmans de l'Université de Ouagadougou impliqués dans l'AEEMB, qui ont « intériorisé ce modèle de domination gérontocratique » (2016: 38). Loin d'être une situation unique au Burkina Faso, les relations intergénérationnelles - souvent conflictuelles - sont au cœur des dynamiques structurant le champ islamique ailleurs en Afrique de l'Ouest en plus d'être étroitement liées aux questions de pouvoir et d'autorité religieuse (Savadogo, LeBlanc et Gomez-Perez 2009; Janson 2010).

\footnotetext{
${ }^{20}$ L'article 37 de la constitution limite tout candidat à deux mandats présidentiels de cinq ans.

${ }^{21}$ En juin et juillet 2013, des dizaines de milliers de personnes avaient manifesté à Ouagadougou et ailleurs au pays. Devant la grogne populaire, le président avait suspendu le processus de mise en place du Sénat.

$22<$ www.burkina24.com/2013/09/14/senat-la-communaute-musulmane-soutien-la-mise-enplace-de-linstitution>

$23<$ https://lefaso.net/spip.php?article55892>
} 
L'après-Compaoré déboucha cependant sur une importante remise en question de la légitimité des représentants de la communauté et de multiples appels publics à des changements en profondeur au sein de la FAIB.

«Plus rien ne sera jamais comme avant » au sein de la FAIB?

Même si une large coalition d'acteurs d'âges, de sexes et de profils socioéconomiques très différents fut à l'origine du soulèvement populaire qui entraina la chute du pouvoir Compaoré en octobre 2014, la mobilisation d'un grand nombre de jeunes burkinabè, à travers des mouvements comme le Balai Citoyen, le Collectif Anti Référendum (CAR) et « Ça Suffit! » ou par l'utilisation des réseaux sociaux, joua un rôle déterminant dans l'insurrection. Hagberg a d'ailleurs parlé de « generational shift » (2015: 115-116) pour qualifier les répercussions de cette révolution. De nombreux jeunes avaient pris conscience de leur force pour faire bouger les choses et conséquemment, plus rien ne pourrait se faire sans les prendre en considération.

Ce changement de mentalité incarné par le slogan «Plus rien ne sera jamais comme avant » eut des retombées importantes dans la communauté musulmane. En effet, un nombre croissant de musulmans, et tout particulièrement des jeunes, remirent publiquement en question la légitimité des ainés qui les représentaient à la FAIB et exigèrent de leur part qu'ils répondent de leur manque d'intégrité pour leur soutien au régime déchu. Les appels pour des réformes en profondeur au sein de la fédération se multiplièrent et le nouveau départ espéré devait s'effectuer par la tenue d'un congrès à la FAIB. Le mandat de ses responsables était expiré depuis 2010 ce qui suscitait d'autant plus d'insatisfaction que suivant la mort de Kanazoé en 2011, la présidence intérimaire, assurée par une rotation entre les membres du présidium, s'éternisait.

La lettre ouverte aux dirigeants de la FAIB écrite par Sidgomdé Yusuf Ouédraogo, qui affirmait se faire le «porte-parole de la jeunesse musulmane » en disant « tout haut ce que beaucoup pensent très bas », alla en ce sens. Bien qu'il s'agisse d'un musulman non connu du milieu associatif islamique, son texte paru en novembre 2014 résumait très bien la frustration partagée par nombre de jeunes interrogés :

[...] gouverner la FAIB par une « présidence mensuelle tournante » ressemble plus à du syndicalisme qu'à de la religion. [...] À quand la date du prochain congrès ? À quand les reformes pour porter au mieux les aspirations des musulmans du Burkina ? [...] la jeunesse musulmane des quatre coins du Burkina 
attend des changements dans le fonctionnement de la FAIB de la même manière qu'elle a contribué quantitativement et qualitativement à la chute du régime Compaoré. [...] Le temps des bénis oui-oui est terminé. Le temps des participations aux colloques pour des perdiems est terminé. Le temps des accointances avec le pouvoir est terminé. (LeFaso.net 30 novembre 2014)

Si la plupart des appels se firent dans des propos plus pondérés, jamais de jeunes musulmans n'avaient critiqué aussi ouvertement le leadership de leurs ainés. D'autres voix se firent également entendre pour reprocher le mutisme et l'inaction des responsables de la FAIB, qui « ont fermé les yeux et ont craint de dénoncer les errements comme l'ont fait les autres confessions qui l'ont fait avec brio ${ }^{24} \gg$ (Le vrai visage de l'islam 5 décembre 2014). Le prêcheur Ismaël Derra, une figure près des courants salafistes et très populaire parmi la jeunesse ouagalaise, avait affirmé en janvier 2015 qu'il fallait revoir la « gouvernance des affaires musulmanes au Burkina Faso » afin de « faire un renouvellement pour un changement tenant compte des aspirations spirituelles des musulmans et de leur positionnement dans les affaires politiques, économiques, sociales » (L'Autre Regard 5 janvier 2015).

Les lignes de fractures de la FAIB, qui furent révélées au grand jour, n'étaient pas seulement générationnelles, mais également régionales. Les élites musulmanes de l'ouest du pays et notamment celles de Bobo-Dioulasso s'étaient senties complètement exclues du processus de création de la fédération (L'Observateur Paalga 23 octobre 2003). Il n'était dès lors pas surprenant que les dirigeants de la Coordination des Associations Islamiques de l'Ouest, un regroupement d'une quarantaine d'organisations musulmanes surtout actives à BoboDioulasso, aient critiqué vertement le leadership des élites de Ouagadougou. Dans une lettre parue en mai 2015, ses responsables reprochaient non seulement les reports continuels d'un nouveau congrès à la FAIB, mais également la concentration des pouvoirs dans la capitale avec l'absence de représentations régionales de la fédération en plus de demander la « démission pure et simple » des dirigeants : «Que vous vouliez tout faire pour toujours vous

\footnotetext{
${ }^{24}$ Par exemple, dans une lettre pastorale signée par tous les évêques du Burkina Faso et publiée en juillet 2013, l’Église catholique affirmait rejeter la mise en place du Sénat et attirait l'attention du gouvernement sur les menaces que son instauration faisait planer sur la paix sociale. Voir Kolesnoré (2016: 18-50).
} 
maintenir à la tête de la FAIB, vous vous trompez d'époque. L'insurrection populaire inch'Allah a ouvert les yeux à tout le monde et surtout aux musulmans. Nous disons fermement aussi que "plus rien ne sera comme avant!”. [...] Non à l'incompétence et à la magouille de toute sorte; Non à la tentative de patrimonialisation de la FAIB » (Sidwaya 7 mai 2015).

Après plusieurs reports, la FAIB tint finalement le congrès tant attendu en juin 2015. À première vue, les décisions qui y furent prises semblaient aller dans le sens d'un « changement dans la continuité » plutôt que des réformes en profondeur. En effet, la présidence tournante entre les quatre membres du présidium fut maintenue. Cependant, leur rôle fut transformé en une sorte de conseil d'administration tandis que les pleins pouvoirs d'exécution allaient dorénavant être confiés au secrétariat exécutif ( $L$ 'Autre Regard 5 juillet 2015). En ce sens, la désignation de Cheikh Sidi Mohamed Koné au poste de secrétaire exécutif national marqua une évolution importante. Titulaire d'une maitrise en sciences économiques et gestion de l'Université de Ouagadougou, il était inspecteur des finances et conseiller à la Cour des comptes au moment de sa sélection. Figure très respectée, il était actif dans le milieu associatif islamique depuis la fin des années 1980 alors qu'il militait à l'AEEMB en plus d'avoir été président du CERFI de 2006 à $2009^{25}$.

Le choix de cet « intellectuel musulman » comme principal porte-parole de la fédération auprès des médias ainsi que lors de rencontres avec des officiels du gouvernement témoignait bien d'une volonté des dirigeants de longue date de la FAIB d'accorder de plus grandes responsabilités à ce profil de musulmans, qui avait jusque-là peiné à faire entendre leur voix. Le maintien du statuquo aurait été intenable comme le souligna le secrétaire général de la Coordination des Associations Islamiques de l'Ouest - cosignataire de la lettre incendiaire que son organisation avait adressé aux leaders de la FAIB - au terme du congrès : « Il n’y a pas eu de questions taboues. [...] Vraiment, les vérités nous nous sommes dit ça face à face

${ }^{25}$ Entretien avec Cheikh Sidi Mohamed Koné, pionnier de l'AEEMB et ancien président du CERFI (2006-2009), Ouagadougou, 21 mai 2015. 
parce que nous disons que, effectivement, l'exemple de ce pays en insurrection, ça a réveillé toutes les consciences, toutes les vérités ${ }^{26}$. »

Outre Cheikh Sidi Mohamed Koné, les nouvelles nominations permirent d'entreprendre un certain virage jeunesse. Par exemple, Mikaïlou Kéré, un ancien militant de l'AEEMB et du CERFI qui avait été directeur de publication de l'ancien mensuel islamique La Preuve (20072011), fut choisi responsable de la communication de la FAIB. La fédération, souvent critiquée à cet égard, s'est montrée passablement active sur Facebook depuis la création d'une page en $2016^{27}$. La FAIB a aussi procédé à l'installation de représentations régionales pour laquelle elle avait été blâmée sévèrement. Ses prises de position inédites à l'occasion des élections de novembre 2015, puis concernant le projet de loi sur les libertés religieuses en janvier 2017 allaient également confirmer que ces changements n'étaient pas seulement cosmétiques. Entretemps, des leaders de l'AEEMB et du CERFI tirèrent profit de la léthargie dans laquelle était plongée la fédération jusqu'à la tenue de son congrès en juin 2015 pour s'impliquer résolument dans le déroulement de la transition au nom de la communauté musulmane.

\section{De « bétail électoral » à force politique? Les musulmans hors de l'arène spirituelle}

Dès les premiers mois qui suivirent l'insurrection d'octobre 2014, des individus des milieux francophones du CERFI et de l'AEEMB occupèrent activement la sphère publique pour tenter de redéfinir la participation citoyenne de la communauté musulmane du pays et, ce faisant, chercher à se poser comme des représentants légitimes de leurs coreligionnaires. D'autres associations islamiques, dont la FAIB, s'engagèrent aussi hors de l'arène spirituelle. Après être restée à l'écart des débats sociopolitiques depuis l'épisode du Sénat, la fédération fit des déclarations très remarquées laissant penser que l'attitude accommodante envers l'État caractérisant historiquement les élites musulmanes burkinabè ne semble plus être autant indéfectible.

Les « intellectuels musulmans » francophones et la promotion d'un islam civil

\footnotetext{
26 ' 1 er congrès ordinaire de la fédération des associations islamiques du Burkina Faso', Radiodiffusion-Télévision du Burkina, 16 juin 2015, 〈https://youtu.be/wsy9M7t9vEo >, consulté le 23 novembre 2018.

27 <www.facebook.com/FAIB.Burkina .
} 
Pour nombre de jeunes « intellectuels » francophones du CERFI et de l'AEEMB, les évènements ayant mené à la chute du président Compaoré illustraient de manière éloquente l'implication insuffisante des leaders de la communauté musulmane dans les grandes questions d'intérêt national. Cet éditorial du bulletin An-Nasr Trimestriel de l'AEEMB, paru en janvier 2015, l'exprimait bien :

Les musulmans individuellement ont activement participé à cette révolution. Mais force est de reconnaître que les musulmans n'ont pas participé au processus en tant que communauté [...]. Nous osons croire que la communauté des musulmans [...] jouera désormais son rôle qui est le leur : éduquer et interpeller les acteurs politiques sur leurs responsabilités à travailler sincèrement pour des populations. (An-Nasr Trimestriel janvier-mars 2015)

Certes, dès les années 1990 et surtout à partir de la décennie suivante, des individus gravitant autour des deux associations avaient tenté de promouvoir l'islam comme structure morale de la pratique citoyenne. Toutefois, leur capacité à influencer véritablement les comportements de leurs coreligionnaires demeura mitigée en raison de leur prudence à dénoncer directement les dérives du régime Compaoré et de leur silence sur les agissements de leurs ainés au centre des mécanismes clientélistes du pouvoir (Madore 2016a: 152-161). Profitant du mutisme observé par les porte-paroles de la FAIB sur le déroulement de la transition et du capital de sympathie qu'ils avaient obtenu pour leur sortie lors de la crise sur le Sénat, des leaders du CERFI et dans une moindre mesure de l'AEEMB s'efforcèrent de mieux positionner leur organisation dans le paysage associatif islamique. Pour ce faire, ils mirent de l'avant leurs différences générationnelles en se présentant comme étant plus aptes que la génération plus âgée à défendre les préoccupations de leur communauté religieuse dans les débats publics. Comme l'ont souligné Masquelier et Soares (2016), se prétendre « jeune » musulman peut être une façon de contester les hiérarchies établies en revendiquant un leadership et une supériorité morale et intellectuelle par rapport à la « vieille » génération.

C'est en ces termes que l'imam Ismaël Tiendrébéogo s'était exprimé dans une entrevue télévisée en 2017. Il avait alors expliqué que les gens de la «vieille génération », qui sont pour l'essentiel des analphabètes ou scolarisés en arabe, « peuvent être facilement instrumentalisés par des intérêts politiques », alors que les jeunes « intellectuels musulmans » francophones ont une « certaine compréhension du contexte [politique] » et « une connaissance des rapports de force », qui « peut permettre de donner un éclairage à cette 
vieille génération ${ }^{28} \gg$. Cela rejoignait les propos d'un ancien militant de l'AEEMB, qui déplorait que « les politiques profitent de la communauté musulmane comme un bétail électoral $^{29}$ ». Dans la même lignée, pour l'imam Tiégo Tiemtoré, les « vieux » d'autres grandes associations islamiques sont «plus malléables » et susceptibles de se laisser prendre « à l'achat de conscience » :

La culture générale politique, ils ne l'ont pas. [...] C'est nous qui l'avons. [...] nous sommes dans un pays pauvre aussi. Au cas où vous tendez 100 000, 200000 à quelqu'un, ça fait l'effet [rires]. [...] Au contraire de l'Église catholique, le cardinal n'a pas besoin de 100 000, 200000 du président. [...] Ils sont à l'abri du besoin. Donc ils sont libres de dire des choses. [...] c'est difficile avec nos vieux, la pauvreté aidant. Mais l'AEEMB/CERFI, Dieu merci, les gens sont des fonctionnaires et des travailleurs ${ }^{30}$.

Le cas de Moussa Nombo, le président du CERFI entre 2009 et 2015, fut particulièrement éloquent pour illustrer l'aspiration de ces musulmans francophones, souvent sans formation formelle dans les sciences islamiques, à se poser en « leader d'opinion » au sein de leur communauté (Triaud 2010). Administrateur des services financiers au ministère de l'Économie et des Finances, son parcours académique entre 1992 et 1997 s'était conclu par l'obtention d'un master en sciences juridiques de l'Université de Ouagadougou. Il se considérait initialement comme étant davantage impliqué comme «syndicaliste » avant d'opter pour la « vie religieuse » en militant pour l'AEEMB. Moussa Nombo y occupa divers postes de responsabilité, puis intégra le CERFI en 2004. Il nous avait expliqué que son arrivée à la tête du CERFI en décembre 2009 marqua un « changement générationnel » au sein de l'association, car outre lui, d'autres jeunes, parmi lesquels se trouvaient d'anciens responsables de l'AEEMB, avaient été affectés au bureau national dans des postes de décision. Parmi les trois grands objectifs stratégiques de son second mandat (2013-2015) se

\footnotetext{
28 'Le Grand déballage avec Imam I. Tiendrebeogo', Burkina Info TV, 11 juillet 2017, <https://youtu.be/IwPvkH0EjUQ>, consulté le 26 novembre 2018.

${ }^{29}$ Entretien avec un ancien militant de l'AEEMB, Ouagadougou, 24 avril 2015.

${ }^{30}$ Entretien avec Tiégo Tiemtoré, imam de l'AEEMB/CERFI, Ouagadougou, $1^{\mathrm{er}}$ mai 2015.
} 
trouvait celui de « faire du CERFI une organisation contribuant à l'éveil de la conscience citoyenne au Burkina Faso par la promotion des valeurs islamiques et républicaines ${ }^{31} »$.

Loin de revendiquer l'hégémonie d'un cadre religieux sur la société, il s'agissait de faire la promotion de formes d'engagement citoyen par le religieux - d'un « islam civil » (Hefner 2011 [2000]) - ou, pour reprendre les termes de Salvatore (2016), d'une « civilité islamique » pouvant favoriser les évolutions démocratiques. Alors que le gouvernement de la transition venait à peine d'être installé, Moussa Nombo et le dirigeant de l'AEEMB avaient fait une déclaration dans laquelle ils avaient appelé les membres du Conseil National de Transition chargé d'organiser les élections présidentielle et législatives de 2015 - à s'attaquer aux problèmes sociaux, à faire preuve d'une « éthique exemplaire dans la gestion des affaires publiques » du pays et à éviter le « favoritisme régionaliste, religieux, corporatiste » (LeFaso.net 28 novembre 2014). En juillet 2015, le président du CERFI avait interpelé l'État sur l'importance du « traitement équitable entre confessions religieuses dans la représentativité au sein des instances décisionnelles et de gouvernance publique » (LeFaso.net 13 juillet 2015). En septembre, il avait également exprimé son « amertume » au premier ministre pour la « gestion sélective des préoccupations confessionnelles » du gouvernement, qui avait ignoré la prière du vendredi dans l'instauration de la journée continue dans la fonction publique (Le Pays 10 septembre 2015).

Tout au long de l'année 2015, cet accent mis sur l'engagement citoyen par Moussa Nombo se traduisit par diverses initiatives, qui bénéficièrent d'une importante couverture médiatique. Le CERFI organisa notamment une activité en juin sur le thème « Intellectuels musulmans : être avec Dieu pour mieux bâtir la cité » avec la présentation d'une table ronde intitulée « Regards croisés sur la situation nationale ». L'évènement, pour lequel nous avions fait de l'observation, avait suscité un véritable engouement tant par le nombre de participants environ deux cents personnes - que par la nature des discussions avec l'assistance, qui avaient été très animées. Les musulmans présents semblaient être déterminés à ce que leur communauté religieuse tire profit du nouveau contexte pour qu'elle pèse enfin dans les débats sociopolitiques, et ce, à la hauteur de son poids démographique.

\footnotetext{
${ }^{31}$ Entretien avec Moussa Nombo, ancien président du CERFI (2009-2015), Ouagadougou, 16 juin 2015 .
} 
Dans son discours d'ouverture, Moussa Nombo avait expliqué que cette activité se voulait une occasion pour les «élites et leaders musulmans »d'échanger sur la situation nationale pour mieux assumer leur fonction d'« éclaireur » au sein de la communauté musulmane. En faisant une allusion voilée aux critiques que la FAIB avait essuyées, il avait affirmé que les musulmans attendent dorénavant des actions de leurs élites qui outrepassent la sphère cultuelle, ce à quoi le CERFI désirait contribuer en s'engageant davantage sur le terrain sociopolitique. Cette rencontre s'inscrivait dans le cadre d'une grande campagne nationale de « sensibilisation citoyenne » auprès des musulmans sur la thématique « spiritualité et citoyenneté à la lumière des enseignements islamiques ». En juillet 2015, les questions de citoyenneté furent aussi le sujet principal du sermon de l'imam Tiégo Tiemtoré à l'occasion de l'Ä̈d el-Fitr (LeFaso.net 20 juillet 2015). Pour la première fois de son histoire, le CERFI s'était également impliqué dans la supervision du scrutin de novembre en fournissant 121 volontaires, préalablement formés par la Commission Électorale Nationale Indépendante (CENI) (LeFaso.net 23 novembre 2015).

S'il est difficile de mesurer l'impact réel de ces différentes initiatives sur la mobilisation citoyenne des musulmans, il n'en demeure pas moins que les « intellectuels musulmans » francophones sont des figures ascendantes dans le champ islamique du pays depuis le soulèvement populaire d'octobre 2014. Les leaders de l'AEEMB et du CERFI bénéficient du fait que la hiérarchie catholique et le Service pastoral pour la formation et l'accompagnement des responsables (SEPAFAR) semblent avoir la volonté de promouvoir ces deux associations comme voix officielle de l'islam burkinabè ${ }^{32}$. En effet, la Jeunesse Étudiante Catholique (JEC) entretient des relations étroites avec l'AEEMB en milieu scolaire et étudiant. Des représentants de l'Église catholique collaborent pour leur part régulièrement avec des membres du CERFI et de l'AEEMB dans le cadre d'activités sur la cohabitation et le dialogue interreligieux.

En outre, les leaders musulmans francophones ont réussi à devenir des intermédiaires entre leur communauté et l'État de plus en plus sollicités au détriment d'autres associations islamiques et de leurs dirigeants, qui occupaient une position beaucoup plus dominante sous le pouvoir Compaoré. Les nombreuses nominations politiques de personnes affiliées à l'AEEMB ou au CERFI par le régime Kaboré sont très évocatrices à cet égard. En avril 2016,

\footnotetext{
${ }^{32}$ Nos remerciements à un des évaluateurs externes pour nous avoir souligné cet élément.
} 
l'imam Ismaël Tiendrébéogo fut choisi président de l'Observatoire National des Faits Religieux (ONAFAR). Moussa Nombo fut, quant à lui, un des deux représentants de la communauté musulmane à la commission constitutionnelle, qui était chargée de la rédaction de l'avant-projet d'une constitution. En 2017, Souleymane Koné, le nouveau président du CERFI (2016-2017), fut nommé ambassadeur au Koweït alors que l'imam Tiégo Tiemtoré fut décoré Chevalier de l'ordre National. Enfin, en octobre 2018, la mosquée du CERFI fut préférée à la grande mosquée de la $\mathrm{CMBF}$, la mosquée de Zangouettin du Mouvement Sunnite ou celle d'Hamdallaye ${ }^{33}$ d'Aboubacar Doukouré pour la tenue de l'« office religieux de la communauté musulmane » dans le cadre de la commémoration de l'insurrection populaire et de la résistance au coup d'État de septembre 2015.

Si ces différentes nominations politiques et reconnaissances symboliques de l'État contribuèrent à légitimer davantage les leaders musulmans francophones, elles pourraient toutefois mettre à mal les prétentions de ces derniers à incarner un islam civil jouant un rôle dans la consolidation de la démocratie. En effet, le régime Kaboré semble vouloir poursuivre le clientélisme utilisé par son prédécesseur, mais en ciblant d'autres associations et dirigeants islamiques. Néanmoins, outre le CERFI et l'AEEMB, plusieurs organisations musulmanes burkinabè, dont la FAIB, commencèrent à leur tour à s'impliquer plus directement dans les débats nationaux du pays pour défendre le bien commun.

La FAIB, de «Commision Lune » à nouvel acteur majeur de la scène politique burkinabè?

Depuis sa création, l'inaction de la FAIB sur le terrain sociopolitique faisait en sorte que beaucoup de musulmans l'assimilaient à une «Commission Lune » servant uniquement à observer les phases lunaires pour annoncer les dates officielles des fêtes islamiques ${ }^{34}$. Un tournant semble toutefois s'être opéré depuis le congrès de 2015 comme en témoigna la publication d'une lettre de la fédération dans la presse et sur sa page Facebook peu de temps avant la présidentielle et les législatives de novembre 2015. La FAIB appelait alors l'ensemble des Burkinabè à participer massivement aux deux scrutins, car « Cet acte serait la

\footnotetext{
${ }^{33}$ Un quartier de Ouagadougou.

${ }^{34}$ Ceci est toutefois à nuancer dans la mesure où les responsables de la FAIB avaient revendiqué une plus grande équité dans le traitement des religions par l'État lors du Conseil Consultatif sur les Réformes Politiques en 2011 et du Forum national sur la laïcité en septembre 2012 .
} 
clef du changement tel que voulu et exprimé par le peuple en octobre 2014 » et ces « voix comptent pour l'atteinte du changement au Burkina Faso, à la lumière de la foi musulmane et des valeurs soutenues et défendues par l'islam » (LeFaso.net 14 novembre 2015). Parmi les quatre critères définissant le choix d'un bon candidat, celui-ci devait avoir « un projet de société clair et réaliste » et prôner « la cohésion sociale et l'équité entre les hommes, au-delà de leur appartenance confessionnelle ». Ce dernier point était particulièrement intéressant dans le contexte du tollé suscité par l'entrevue d'Ablassé Ouédraogo dans le magazine Jeune Afrique quelques mois auparavant ${ }^{35}$.

Cette déclaration publique de la FAIB, qui s'ajoutait à celle de la Coordination des Associations Islamiques de l'Ouest en septembre 2015 dressant une liste pléthorique de « préoccupations des musulmans au Burkina Faso » à l'intention de la classe politique qui était prévenue qu'ils seraient «très regardants sur leur vote »(LeFaso.net 13 septembre 2015), étaient totalement inédites dans leur forme d'expression. En effet, historiquement, les principaux leaders musulmans du pays se limitaient plutôt à de simples bénédictions pour le déroulement d'élections apaisées sans véritablement chercher à exprimer formellement les doléances de la communauté ou à mobiliser leurs coreligionnaires à participer aux scrutins (Le Pays 4 novembre 2005; Sidwaya 17 novembre 2010).

Le projet de loi sur les libertés religieuses illustra aussi le changement qui est en train de s'opérer au sein de la FAIB. Lors du conseil des ministres du 30 novembre 2016, le gouvernement annonça les premiers détails de cette loi, qui devait combler le vide juridique entourant la définition et l'application effective du principe de laïcité par l'État notamment la réglementation encadrant la construction de lieux de culte et la création d'associations confessionnelles (Le Pays $1^{\mathrm{er}}$ décembre 2016). Le projet de loi devait être examiné à l'Assemblée nationale au cours d'une session extraordinaire en janvier 2017. Le 7 janvier, la FAIB fit paraitre un long communiqué de presse pour faire part de ses « inquiétudes profondes sur certaines dispositions du texte », qui pourraient « réduire la liberté de

\footnotetext{
${ }^{35}$ Candidat à l'élection présidentielle, il avait présenté ses « trois atouts principaux », qui lui donnaient à son avis de bonnes chances de l'emporter : « Je suis Moagha du plateau central, et les Mossis sont une forte composante du Burkina Faso. Je suis aussi musulman, ce qui n'est pas rien dans un pays où $70 \%$ des gens le sont également [...] (Jeune Afrique 8 juin 2015).
} 
conscience et de culte » et avoir « très probablement des conséquences dommageables sur le vivre ensemble »(LeFaso.net 7 janvier 2017). Parmi les dix articles jugés problématiques et largement commentés dans le document se trouvaient entre autres les craintes concernant l'interdiction possible des rassemblements pour la prière sur le bord des routes; des espaces formels ou non de prière dans les services de l'État ; du port du voile dans l'administration publique ; de prier et de porter des vêtements religieux au travail pour les enseignants musulmans ; des activités organisées par les associations d'élèves et d'étudiants musulmans dans les établissements publics. La FAIB avait conclu sa déclaration en appelant au retrait du projet de loi.

De nombreux musulmans saluèrent grandement cette sortie de la FAIB, jugeant qu'elle avait bien défendu les intérêts de la communauté. Sur la page Facebook de la fédération, la publication avait d'ailleurs été partagée à près de 1000 reprises en plus d'avoir été commentée par plus de 500 personnes $^{36}$, sans commune mesure avec l'engagement habituel. Devant cette polémique, le ministre d'État et ministre de l'Administration Territoriale, de la Décentralisation et de la Sécurité Intérieure, discuta avec une délégation de la FAIB deux jours plus tard pour les informer que le gouvernement avait décidé de retirer le projet de loi. Le 11 janvier, des représentants de l'Union pour le Progrès et le Changement (UPC), le principal parti d'opposition, rencontrait les responsables de la FAIB pour manifester leur soutien. Enfin, peu de temps après, c'était au tour du président Kaboré de s'entretenir avec des dirigeants de la fédération sur le projet de loi. Au cours de cet échange, Cheikh Sidi Mohamed Koné avait affirmé souhaiter l'application d'« une laïcité qui puisse garantir la paix entre les différentes communautés religieuses de ce pays » en plus d'évoquer auprès du chef de l'État les difficultés auxquelles sont confrontés les établissements d'enseignement islamique et leur désir que les musulmans soient entendus dans le processus de rédaction de la nouvelle constitution (FasoZine 17 janvier 2017).

Par le passé, l'État burkinabè avait certes déjà dû faire marche arrière à quelques occasions devant le mécontentement des musulmans notamment en ce qui concerne son implication dans l'organisation du hadj. Cependant, la rapidité avec laquelle le gouvernement recula dans

\footnotetext{
36 'Communiqué FAIB relatif au projet de loi sur les libertés religieuses au Burkina', FAIB, 7 janvier 2017, <www.facebook.com/FAIB.Burkina/posts/255830204837153>, consulté le 15 janvier 2017.
} 
le dossier du projet de loi sur les libertés religieuses et la façon dont plusieurs politiciens, tant du pouvoir que de l'opposition, cherchèrent à ménager les sensibilités des leaders de la FAIB illustraient bien un potentiel changement de paradigme, qui s'était amorcé depuis la chute de Blaise Compaoré, alors que les élites musulmanes avaient pratiquement toujours offert un soutien inconditionnel au pouvoir.

\section{Conclusion}

Pour Zaman (2002), l'ampleur des défis auxquels sont confrontés les oulémas dans le monde musulman contemporain est sans précédent alors que leur autorité religieuse est notamment contestée par de nouveaux intellectuels éduqués dans des universités occidentalisées et souvent dépourvus de formation formelle en sciences islamiques. Toutefois, comme il l'a souligné, de nombreux théologiens ont malgré tout su s'adapter pour préserver, voir renforcer leur position. Au Burkina Faso, de grandes figures religieuses ayant étudié dans de prestigieuses universités du monde arabe exercent toujours une forte influence dans le paysage religieux. En effet, les sermons et prédications salafistes d'Ismaël Derra (Université al-Azhar), de Mohamad Kindo (Université de Médine) et de Mohamad Sawadogo (Université al-Imam Muhammad ibn Saoud de Riyad) trouvent un écho important auprès d'une partie des jeunes musulmans en plus d'être largement diffusés sur la radio islamique Al Houda (Savadogo et Gomez-Perez 2011) et sur Internet (Madore 2016c; Madore et Audet Gosselin 2019). Les confréries soufies des chefs religieux tijâni Aboubacar Doukouré (Université de Médine) et Aboubacar Maïga II (Université al-Azhar) rejoignent aussi un très grand nombre de fidèles (Dassetto, Laurent et Ouédraogo 2012; Vitale 2012). Ceci est d'autant plus vrai que si le français est devenu une langue de plus en plus légitime pour propager l'islam, les langues nationales restent primordiales pour rallier un vaste public non francophone maitrisant le mooré, le dioula ou le fulfuldé.

Bien que les « intellectuels musulmans » francophones ne soient pas parvenus à se substituer aux élites religieuses traditionnelles, il n'en demeure pas moins qu'ils ont participé activement aux transformations significatives qui sont à l'œuvre au sein de la communauté musulmane du Burkina Faso depuis le départ du président Compaoré en octobre 2014. Ils ont su profiter du nouveau contexte politique pour se positionner plus avantageusement dans la concurrence que se livrent des acteurs islamiques dans la sphère publique pour le leadership religieux. Des figures francophones du CERFI et de l'AEEMB ont cherché à se différencier de l'« ancienne génération »-critiquée pour sa proximité avec le précédent régime -, en se 
présentant comme les principaux vecteurs d'un islam civil, qui accompagnerait les évolutions démocratiques du pays. S'il est encore trop tôt pour conclure à des changements durables, la question de la participation citoyenne des musulmans n'avait jamais été traitée à ce point par des structures islamiques et leurs dirigeants dans l'histoire du pays.

Les connaissances religieuses demeurent certes essentielles, mais la légitimité et l'autorité des responsables d'organisations musulmanes au Burkina Faso découlent de plus en plus de la capacité à s'engager dans la sphère publique pour débattre des notions de « bien commun » à la lumière de l'islam et à défendre les aspirations de leurs coreligionnaires. Ceci est d'autant plus important pour s'affirmer dans le champ hautement concurrentiel des associations islamiques et revendiquer la possibilité de s'exprimer au nom de la « communauté »avec l'émergence d'une sphère publique islamique un peu partout en Afrique de l'Ouest (Launay et Soares 1999; Holder 2009). Alors que le discours médiatique sur le djihad au Sahel des dernières années a contribué à alimenter les inquiétudes sur l'islam et la politique dans la région, les développements récents au Burkina Faso rappellent que nombre de réalités caractérisant ces communautés musulmanes, qui sont moins couvertes, mais plus prégnantes, sont souvent éclipsées par les manifestations de violence. La participation accrue des musulmans dans l'arène politique ne doit donc pas être comprise comme la montée d'un « islamisme » au Burkina Faso, mais l'« apolitisme » historiquement observé par ses élites semble être une chose du passé.

\section{Remerciements}

Mes remerciements aux trois évaluateurs externes qui m'ont permis de renforcer grandement l'argumentation du texte. La recherche sur laquelle s'appuie cet article a été financée par le Conseil de recherches en sciences humaines (CRSH) du Canada.

\section{Bibliographie}

Audet Gosselin, L. (2016) 'Une nation pluraliste? Les limites du dialogue interconfessionnel chez les jeunes militants religieux à Ouagadougou', Canadian Journal of African Studies / Revue canadienne des études africaines 50 (1): 105-26.

Bamyeh, M. A. et A. Salvatore (2017) 'The role of intellectuals within late-colonial and postcolonial public spheres' in A. Salvatore, R. Tottoli et B. Rahimi (eds), The Wiley Blackwell History of Islam. Hoboken: John Wiley \& Sons. 
Bouron, J.-M. (2011) ‘Amitiés, inimitiés. Les rapports incertains de l'Église catholique avec la Première République voltaïque (1960-1966)', Civilisations 60 (1): 123-42.

Camara, E. H. M. S. et M. Bodian (2016) 'Islam in the academic sphere in Senegal: the case of Cheikh Anta Diop University in Dakar (UCAD)', Contemporary Islam 10 (3): 379-98.

Cissé, I. (1994), 'Islam et État au Burkina Faso : de 1960 à 1990'. thèse de doctorat, Université de Paris VII - Denis Diderot.

Cissé, I. (2009) 'Le wahhabisme au Burkina Faso : dynamique interne d'un mouvement islamique réformiste', Cahiers du CERLESHS 24 (33): 1-33.

Cissé, I. (2010) 'El hadj Oumarou Kanazoé : homme d’affaires et mécène dans la communauté musulmane burkinabè', Cahiers du CERLESHS 25 (34): 151-86.

Cissé, I. (2015) 'Islam et économie au Burkina Faso. Relations et enjeux', Islam et sociétés au sud du Sahara 4: 9-28.

Dassetto, F., P.-J. Laurent et T. Ouédraogo (2012) Un islam confrérique au Burkina Faso : actualité et mémoire d'une branche de la Tijâniyya. Paris: Karthala.

Eickelman, D. F. et J. P. Piscatori (2004) Muslim Politics, 2e éd. Princeton: Princeton University Press.

Fancello, S. (2007) 'Les défis du pentecôtisme en pays musulman (Burkina Faso, Mali)', Journal des africanistes 77 (1): 29-53.

Frégosi, F. (2004) 'L'Imam, le conférencier et le jurisconsulte : retour sur trois figures contemporaines du champ religieux islamique en France', Archives de sciences sociales des religions 125: 131-46.

Gruénais, M.-É. (1985) ‘Aînés, aînées; cadets, cadettes. Les relations aînés/cadets chez les Mossi du centre (Burkina Faso)' in M. Abélès et C. Collard (eds), Âge, pouvoir et société en Afrique noire. Paris: Karthala.

Hagberg, S. (2015) “"Thousands of New Sankaras”: resistance and struggle in Burkina Faso', Africa Spectrum 50 (3): 109-21. 
Harsch, E. (2017) Burkina Faso: a history of power, protest, and revolution. London: Zed Books.

Hefner, R. W. (2011 [2000]) Civil Islam: Muslims and democratization in Indonesia. Princeton: Princeton University Press.

Hilgers, M. et A. Loada (2013) 'Tensions et protestations dans un régime semi-autoritaire : croissance des révoltes populaires et maintien du pouvoir au Burkina Faso', Politique africaine 131 (3): 187-208.

Holder, G. (ed) (2009) L'islam, nouvel espace public en Afrique. Paris: Karthala.

Idrissa, R. (2017) The Politics of Islam in the Sahel: between persuasion and violence. New York: Routledge.

Janson, M. (2010) 'The battle of the ages: contests for religious authority in the Gambia' in L. Herrera et A. Bayat (eds), Being Young and Muslim: new cultural politics in the global South and North. Oxford: Oxford University Press.

Kane, I. (2016) “"Les catholiques sont l'élite!” : représentations de l’espace politique par une minorité religieuse au Burkina Faso', Canadian Journal of African Studies / Revue canadienne des études africaines 50 (1): 65-86.

Kobo, O. (2012) Unveiling Modernity in Twentieth-century West African Islamic Reforms. Leiden: Brill.

Kouanda, A. (1998) 'Les conflits au sein de la Communauté musulmane du Burkina : 19621986' in O. Kane et J.-L. Triaud (eds), Islam et islamismes au sud du Sahara. Paris: Karthala. Krämer, G. et S. Schmidtke (eds) (2006) Speaking for Islam: religious authorities in Muslim societies. Leiden, Brill.

Langewiesche, K. (2011) 'Le dialogue interreligieux au service du développement : élites religieuses et santé publique au Burkina Faso', Bulletin de l'APAD 33:

$<$ https://journals.openedition.org/apad/4087>.

Launay, R. et B. F. Soares (1999) 'The formation of an "Islamic sphere" in French colonial West Africa', Economy and Society 28 (4): 497-519. 
Laurent, P.-J. (2009 [2003]) Les pentecôtistes du Burkina Faso : mariage, pouvoir et guérison. Paris: Karthala.

Madore, F. (2016a) La construction d'une sphère publique musulmane en Afrique de l'Ouest. Québec/Paris: Presses de 1’Université Laval/Hermann.

Madore, F. (2016b) 'Islam, médias, mise en place du Sénat et article 37 de la Constitution : changement de paradigme au Burkina Faso (1991-2014)?', Canadian Journal of African Studies / Revue canadienne des études africaines 50 (1): 7-27.

Madore, F. (2016c) 'L'islam ivoirien et burkinabé à l'ère du numérique 2.0', Journal des anthropologues 146-147: 151-78.

Madore, F. et L. Audet Gosselin (2019) 'Le religieux sur Internet et dans les NTIC au Burkina Faso' in A. Degorce, L. O. Kibora et K. Langewiesche (eds), Rencontres religieuses et dynamiques sociales au Burkina Faso. Dakar: Amalion.

Mandaville, P. (2007) 'Globalization and the politics of religious knowledge: pluralizing authority in the Muslim world', Theory, Culture \& Society 24 (2): 101-15.

Masquelier, A. et B. F. Soares (2016) 'Introduction' in A. Masquelier et B. F. Soares (eds), Muslim Youth and the 9/11 Generation. Albuquerque: University of New Mexico Press.

Mazzocchetti, J. (2009) Être étudiant à Ouagadougou : itinérances, imaginaire et précarité. Paris: Karthala.

Miran-Guyon, M. et N. Oyewolé (2015) 'Côte d'Ivoire, un islam d'expression française à la fois tangible et restreint', Histoire, monde et cultures religieuses 36 (4): 141-58.

Nsaibia, H. (2018) 'The fledgling insurgency in Burkina's East', Armed Conflict Location \& Event Data Project (ACLED): <www.acleddata.com/2018/09/20/the-fledgling-insurgency-inburkinas-east>.

Nsaibia, H. et W. Caleb (2018) 'Ansaroul Islam and the growing terrorist insurgency in Burkina Faso', CTC Sentinel, 11 (3): 21-6. 
Otayek, R. (1984) 'La crise de la communauté musulmane de Haute-Volta. L'islam voltaïque entre réformisme et tradition, autonomie et subordination', Cahiers d'études africaines 24 (3): 299-320.

Otayek, R. (1996) 'L'islam et la révolution au Burkina Faso : mobilisation politique et reconstruction identitaire', Social Compass 43 (2): 233-47.

Otayek, R. (1997) 'L’Église catholique au Burkina Faso. Un contre-pouvoir à contretemps de l'histoire?' in F. Constantin et C. Coulon (eds), Religion et transition démocratique en Afrique. Paris: Karthala.

Ouédraogo, Y. (2019) 'Le réformisme islamique francophone au Burkina Faso' in A. Degorce, L. O. Kibora et K. Langewiesche (eds), Rencontres religieuses et dynamiques sociales au Burkina Faso. Dakar: Amalion.

Saint-Lary, M. (2011) 'Le Coran en cours du soir. La formation comme outil de réislamisation des musulmans francophones', Ethnographiques.org 22: <www.ethnographiques.org/2011/Saint-Lary>.

Salvatore, A. (2016) The Sociology of Islam: knowledge, power and civility. Oxford: WileyBlackwell.

Salvatore, A. et D. F. Eickelman (2006) 'Preface: public Islam and the common good' in A. Salvatore et D. F. Eickelman (eds), Public Islam and the Common Good. Leiden: Brill.

Samson, F. (2018) 'Pluralisme et concurrence islamique dans l'appropriation d'un espace public religieux. Analyses comparées au Sénégal et au Burkina Faso’ in G. Holder et J.-P. Dozon (eds), Les politiques de l'islam en Afrique. Mémoires, réveils et populismes islamiques. Paris: Karthala.

Savadogo, M. et M. Gomez-Perez (2011) 'La médiatisation des prêches et ses enjeux. Regards croisés sur la situation à Abidjan et à Ouagadougou', Ethnographiques.org 22: <www.ethnographiques.org/2011/Savadogo-Gomez-Perez>.

Savadogo, M., M.-N. LeBlanc and M. Gomez-Perez (2009) 'Young men and Islam in the 1990s: rethinking an intergenerational perspective', Journal of Religion in Africa 39 (2): 186218. 
Sawadogo, K., B. Gansonré et M. Ouibga (2016), Histoire du CERFI des origines à 2015. Ouagadougou: ESIF Sarl.

Somé, M. (2001) 'Christian base communities in Burkina Faso: between church and politics', Journal of Religion in Africa 31 (3): 275-304.

Sounaye, A. (2015) 'Le français : langue d'élite, langue de religiosité, outil de réislamisation au Niger', Histoire, monde et cultures religieuses 36 (4): 119-40.

Triaud, J.-L. (2010) 'Les nouveaux intellectuels islamiques francophones. Autour de deux colloques', Islam et société au sud du Sahara 2: 55-76.

Vanvyve, A. (2015) 'L'islam burkinabé sous la IV ${ }^{\mathrm{e}}$ République', Cahiers d'études africaines 219 (3): 489-508.

Vanvyve, A. (2016) 'L'érosion de l'autorité musulmane à Ouagadougou : le discours de militants de l'Association des Elèves et Etudiants Musulmans au Burkina', Canadian Journal of African Studies / Revue canadienne des études africaines 50 (1): 29-44.

Vitale, M. (2012) 'Trajectoires d'évolution de l'islam au Burkina Faso', Cahiers d'études africaines 206-207 (2): 367-87.

Zaman, M. Q. (2002) The Ulama in Contemporary Islam: custodians of change. Princeton: Princeton University Press.

Articles de journaux, publications islamiques et sites Internet

'1er congrès ordinaire de la fédération des associations islamiques du Burkina Faso', Radiodiffusion-Télévision du Burkina, 16 juin 2015, 〈https://youtu.be/wsy9M7t9vEo>, consulté le 23 novembre 2018.

'Ablassé Ouédraogo : “J'ai toutes mes chances à la présidentielle d'octobre", Jeune Afrique, 8 juin 2015, <www.jeuneafrique.com/234008/politique/burkina-ablass-ou-draogo-j-ai-toutesmes-chances-la-pr-sidentielle-d-octobre/>, consulté le 29 septembre 2017.

'CERFI : la foi et le reste', L’Observateur Paalga, 5 février 1997.

'Cheikh Ismaël Derra : "Beaucoup de responsables musulmans ne méritent pas leur place", L'Autre Regard 22, 5 janvier-5 février 2015. 
'Communiqué FAIB relatif au projet de loi sur les libertés religieuses au Burkina', FAIB, 7 janvier 2017, <www.facebook.com/FAIB.Burkina/posts/255830204837153>, consulté le 15 janvier 2017.

'Conseil des ministres : pour un encadrement de l'exercice des libertés de religion', Le Pays, $1^{\text {er }}$ décembre 2016.

'Déclaration des Associations islamiques du Burkina Faso sur la situation nationale', L’Observateur Paalga, 4 janvier 1999.

'Élections 2015 : la communauté musulmane définit les critères d'un bon choix', LeFaso.net, 14 novembre 2015, <https://lefaso.net/spip.php?article68019>, consulté le 14 novembre 2015.

'Élections du 29 novembre 2015 : le CERFI déploiera 121 superviseurs', LeFaso.net, 23 novembre 2015, <http://lefaso.net/spip.php?article68214>, consulté le 24 novembre 2015.

'Enquête démographique par sondage en République de Haute-Volta 1960-1961 (Tome I)', 〈www.odsef.fss.ulaval.ca/sites/odsef.fss.ulaval.ca/files/fonds_gp/c-doc_365_odsef.pdf>, consulté le 29 janvier 2018.

'Forum des musulmans : des jalons d'une unité organisationnelle', Sidwaya, 19 juin 2001.

'Imam Khalidou Ilboudo : "Nous devons refonder notre politique de la FAIB"', L'Autre Regard 28, 5 juillet-5 août 2015.

'Insurrection populaire au Burkina : le message conjoint du CERFI et de l'AEEMB', LeFaso.net, 28 novembre 2014, <https://lefaso.net/spip.php?article62037>, consulté le 30 novembre 2014.

'Journée continue à la fonction publique : le CERFI dénonce une non-prise en compte des préoccupations des musulmans', Le Pays, 10 septembre 2015.

'La Coordination de l'Ouest exige la démission du bureau fédéral', Sidwaya, 7 mai 2015.

'Le Grand déballage avec Imam I. Tiendrebeogo', Burkina Info TV, 11 juillet 2017, <https://youtu.be/IwPvkH0EjUQ>, consulté le 26 novembre 2018. 
'Lettre ouverte au Présidium de la Fédération des associations islamiques du Burkina', LeFaso.net, 30 novembre 2014, <https://lefaso.net/spip.php?article62053>, consulté le 30 novembre 2014.

'Liberté religieuse : après le retrait du projet de loi, la FAIB est satisfaite et le dit à Roch Kaboré', FasoZine, 17 janvier 2017, <www.fasozine.com/actualite/politique/662-libertereligieuse-apres-le-retrait-du-projet-de-loi-la-faib-exprime-sa-satisfaction-a-rochkabore.html>, consulté le 19 avril 2017.

'Lutte contre l'homosexualité au Faso : les musulmans de l'Ouest haussent le ton', LeFaso.net, 13 septembre 2015, <https://lefaso.net/spip.php?article66830>, consulté le 14 septembre 2015 .

'Lutte contre le terrorisme : le cheval de bataille du CERFI', Le Pays, 2 avril 2016.

'Musulmans du Burkina : pas de fédération restrictive', L'Observateur Paalga, 23 octobre 2003.

'Musulmans et chrétiens ont prié pour une élection apaisée', Sidwaya, 17 novembre 2010.

'Nord du Burkina Faso : ce que cache le jihad', International Crisis Group, 12 octobre 2017, <www.crisisgroup.org/fr/africa/west-africa/burkina-faso/254-social-roots-jihadist-violenceburkina-fasos-north>, consulté le 11 janvier 2018.

'Projet de loi sur les libertés religieuses : les associations islamiques demandent le retrait', LeFaso.net, 7 janvier 2017, <https://lefaso.net/spip.php?article75098>, consulté le 8 janvier 2017.

'Ramadan 2015 : “Épargner le Burkina des images désastreuses et poignantes de populations faméliques, traversant les frontières...", imam Tiégo Tiemtoré', LeFaso.net, 20 juillet 2015, $<$ https://lefaso.net/spip.php?article65929>, consulté le 22 juillet 2015.

'Ramadan : la présidentielle au cœur des prières', Le Pays, 4 novembre 2005.

'Ramadan : rupture collective du jeûne avec l'ambassadeur de France au Burkina', LeFaso.net, 13 juillet 2015, <https://lefaso.net/spip.php?article65812>, consulté le 13 juillet 2015. 
'Recensement général de la population et de 1'habitation au Burkina Faso (RGPH) en 2006',

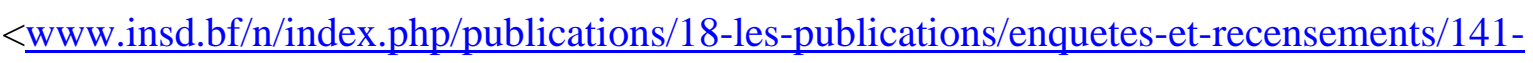
recensement-general-de-la-population-et-de-l-habitation-au-burkina-faso-rgph-en-2006>, consulté le 20 janvier 2018.

'Récépissé de déclaration d'association n²006-078/MATD/SG/DGLPAP/DOASOC du 07 mars 2006', <www.legiburkina.bf/m/Sommaires_JO/Récépissé_MATD_2006_00078.htm>, consulté le 25 novembre 2017.

'Situation politique du Burkina Faso : le rôle de la communauté des musulmans', An-Nasr Trimestriel 54, janvier-mars 2015.

'Soutien à Blaise Compaoré : la FEDAP-BC pour mieux canaliser les forces', Sidwaya, 16 octobre 2007, <https://lefaso.net/spip.php?article24004>, consulté le 24 novembre 2017.

'Vie politique nationale : la FAIB muette comme toujours', Le vrai visage de l'islam 21, 5 décembre 2014-5 janvier 2015.

'Ziniaré : AG statutaire de la communauté musulmane', Sidwaya, 24-25 décembre 1997.

\section{Résumé}

L'attention portée sur les menaces sécuritaires qui planent sur le Burkina Faso a éclipsé les transformations sous-jacentes qui sont en train de s'opérer dans le paysage associatif islamique du pays depuis le départ du président Blaise Compaoré en octobre 2014. Ce bouleversement politique a eu des conséquences significatives sur la participation des musulmans dans les débats sociopolitiques, les dynamiques intergénérationnelles et, plus largement, les bases sur lesquelles l'autorité religieuse est revendiquée. Cet article propose donc d'analyser la concurrence que se livrent les acteurs islamiques dans la sphère publique pour le leadership religieux en se focalisant principalement sur la perspective des « intellectuels musulmans » francophones. L'étude montre, d'une part, que le fossé croissant entre la gérontocratie aux commandes des grandes associations musulmanes et les jeunes, qui était jusque-là demeuré à l'état latent, s'est révélé avec force suivant l'insurrection populaire. D'autre part, profitant de l'espace laissé vacant par les porte-paroles traditionnels de la communauté durant le processus de transition, certains jeunes « intellectuels musulmans » francophones ont cherché activement à se présenter comme les véhicules d'un « islam civil » 
en promouvant de nouvelles formes d'engagement citoyen par le religieux afin de se positionner plus avantageusement dans le champ islamique. 